\title{
AN OBSERVATIONAL STUDY OF THE INTERACTION OF IMPULSIVE AND PERIODIC PERTURBATIONS IN THE SOLAR ATMOSPHERE
}

\author{
F.-L. DEUBNER AND R. KLEINEISEL \\ Astronomisches Institut \\ Am Hubland, D-97074 Würzburg
}

\begin{abstract}
Time series of simultaneous high resolution filtergram observations in white light and in $\mathrm{CaK}_{2}$ have been employed to study the dynamical interdependence of the 'low' and the 'high' layers of the solar atmosphere, the 3-D propagation of perturbations in the stratification, and the excitation of p-mode and other oscillations. Impulsive and oscillatory perturbations have been isolated in the wavenumber and frequency regime, and their mutual interaction was also studied.

Several of our findings challenge interpretation and deserve further study.

- The occurrence of a granule can be clearly recognized in the upper chromosphere.

- A granule induces low brightness and low oscillation amplitudes at the $\mathrm{CaK}_{2}$ level, whereas the opposite happens at the intergranular border.

- It is not always evident that the 'source' of the perturbation is located at the lower, i.e. the photospheric layer.

- It is far from being clear which components of the various perturbations contribute to a net outward mechanical energy flux.
\end{abstract}

\section{Introduction}

Stochastic excitation of p-modes by turbulent convection is generally considered a viable and satisfactory model of the origin of the 5-min oscillations of the sun (cf. e.g. Kumar et al., 1990). This process incorporates the explanation of the pseudo p-modes that occur at frequencies, where resonant eigenmodes of the global p-mode cavity do not exist (Kumar, 1993).

How the energy is transferred to the wave field from individual convective eddies (granules, downdrafts etc.), however, is not being considered 
in detail in this model. Also, observations that could help treating this problem quantitatively are scarce, and are scattered irregularly over more than 30 years that elapsed since the first detailed spatio-temporal studies of solar p-modes were performed. Even taking the difficulty of obtaining observations from the ground with sufficient spatial resolution (preferably $\lesssim 1$ arc sec) during extended periods of time $(\gtrsim 20 \mathrm{~min})$ into account, the material available makes a rather casual impression, and progress towards a more comprehensive treatment of the subject appears to be lacking.

Two paradigms have been drawn up in the course of this long interval since Evans and Michard (1962) first discussed the vertical progression of a photospheric perturbation (notably a granule) into the solar atmosphere.

\section{Paradigm \#1: The granular piston}

According to the description of this event given by Evans and Michard, the initial brightening of a granule is followed first by upward motion, and then by regular oscillations, at successively higher layers in the atmosphere (cf. also Fig. 2 in Noyes, 1967, for illustration). Since the period of oscillation in the upper part of the atmosphere is shorter than below, the initial phase lag of the upper layers is quickly compensated, and the whole atmosphere swings in phase before the motion decays. A rough estimate of the speed of propagation of the initial perturbation yields the value of the local sound velocity.

The hydrodynamic problem of describing the motions excited by a piston driven from below into an isothermal atmosphere overlaid by a hot corona was solved analytically in 2-D by Meyer and Schmidt (1967). The good qualitative agreement with the observations lent strong support to the long held belief that in general the 5 -min oscillations were primarily a local phenomenon. Much later, Goode et al. (1992) presented a numerical calculation of a rather similar process in a realistic solar atmosphere in 1-D. Evidently, the evolution of the perturbation with height in the atmosphere (cf. Fig. 1 of this paper) has a high degree of likeness with the Figure in Noyes (1962). Unfortunately, the horizontal dimension is missing in this calculation.

As a first observational approach of the two-dimensional problem, Deubner (1975) has selected from a 32 min time series of the evolution of a 320 arc sec wide region at the center of the disk, observed simultaneously in four spectral lines, the one event characterized by the highest amplitude of vertical oscillatory motions in the $3 \mathrm{mHz}$ range. The analysis of this particular event did not only corroborate the granular piston paradigm in displaying p-mode oscillations with decreasing period at increasing height on top of a prominent granule. It also shows long period g-modes excited at the flanks of the granule as predicted by Meyer and Schmidt, as a consequence of the 
lateral propagation of the perturbation at higher layers.

With regard to spectral intensity, the picture in this observation is much less clear; but that was not a subject of the Meyer and Schmidt paper either.

\section{Paradigm \#2: The intergranular downdraft}

The source of oscillations in this paradigm is thought to be turbulent motion created within the downdraft funnel by high horizontal velocity gradients giving rise to high frequency sound waves that appear in the photosphere.

This idea was checked observationally by Deubner and Laufer (1983) who used a similar data set as in the 1975 paper, selecting this time 132 events with increased power in the $11-45 \mathrm{mHz}$ range. The averaged temporal sequence clearly shows at the center the signature of a downdraft with reduced brightness at the photospheric level about 2 min before the high frequency event. Also in the low chromosphere $(\mathrm{NaD})$, downward motion is associated with this scenario.

Observational evidence was further elaborated by Rimmele et al. (1995) who found in a 1-D analysis of spatial high resolution data that the temporally averaged high frequency acoustic flux is inversely correlated with the normalized intensity in the Fe 5434 line wing at $+20 \mathrm{~m} \AA$ from the line center. Moreover it was confirmed that the flux reaches a maximum about 2 min after minimum brightness in the continuum. Espagnet et al. (1996) documented the association of intergranular darkening and prominent oscillations in the 5-min range by applying to one and the same set of 2-D data two different $\mathrm{k}-\omega$ filters that isolate the oscillatory from the convective regime.

Looking at this observational material, it has been our impression so far, that the two paradigms are not really contradictory; rather they seem to emphasize different aspects of the interaction between granulation and oscillations. Obviously the goal of future observations had to be to fully deploy the 2-D aspect of the local phenomena as well as to provide insight into the height dependence of the perturbation. Another important aspect in view of the omnipresence of the high amplitude global p-modes is the necessity to acquire a sufficient volume of statistically independent data in order to sift the local response from that intrusive oscillatory background.

\section{Observations}

The new observational material discussed in the following section consists of a 2.5 hours time series of filtergrams taken simultaneously in the continuum at $5000 \AA$, and with a $0.6 \AA$ passband centered on CaK, with the German VTT of the Teide Observatory on Tenerife. The original field of view in the 
quiet center of the disk is $180 \times 180$ arc sec, corresponding to $1024 \times 1024$ pixels of the CCD arrays. The exposure time was $1 \mathrm{sec}$, at a $12 \mathrm{sec}$ cadence. The seeing conditions varied from excellent to good.

To separate the 'convective' from the 'oscillatory' components of the observed brightness fluctuations we have applied two different spatio-temporal filters similar to those described in Espagnet et al. (1996). A 'subsonic' filter transmits all fluctuations with a ratio $\nu / k_{H} \leq 6.5 \mathrm{~km} \mathrm{~s}^{-1}$ corresponding to the velocity of horizontally propagating sound waves; it transmits brightness fluctuations due to gravity waves as well as convective perturbations. The other filter is a band pass transmitting frequencies from $2 \mathrm{mHz}$ to 10 $\mathrm{mHz}$ with a limiting ratio of $\nu / k_{H} \geq 9 \mathrm{~km} \mathrm{~s}^{-1}$; there we find evanescent and propagating $\mathrm{p}$-modes in the observed range of frequencies. In addition, a time dependent r.m.s. amplitude of the oscillatory component was defined for each picture element with the help of the band pass filtered data set, using a 144 sec running mean.

After filtering the data we have selected four data cubes with a volume of $12.7 \mathrm{Mm} \times 12.7 \mathrm{Mm} \times 20 \mathrm{~min}$ each, to search for suitable reference locations, or 'starting points', for the subsequent spatio-temporal analysis, taking the distribution of several scattered fragments of the chromospheric network into account. We have defined a total of 256 starting points by finding the 128 brightest and the 128 darkest pixels that are both spatially and temporally unrelated, within the 'subsonic' continuum cube.

Centered around these starting points we have finally averaged the evolution of the brightness distribution both azimuthally and over all 128 events, covering a radial range of $7.6 \mathrm{Mm}$, and a time interval of $600 \mathrm{sec}$ before and $600 \mathrm{sec}$ after maximum (granule) or minimum (intergranule) brightness in the continuum. Figures 1 and 2 display the average evolution of brightness and r.m.s. amplitude in continuum light and in the light of the CaK line.

\section{Results and discussion}

Inspecting Figure 1, we shall discuss what appears relevant for an improved understanding of the convection - oscillation interaction.

Testing Paradigm \#1 (granule events): In white light, i.e. in the photosphere, we observe that the occurrence of a granule is quickly followed by a substantial decrease of oscillatory power ( $10 \%$ to $15 \%$ below average) lasting about $600 \mathrm{sec}$. The extent of the region with reduced power remains essentially confined within a constant area during this interval, and does not spread beyond the size of a granule. We note that the granular event is preceded by a maximum of oscillatory power about $300 \mathrm{sec}$ before the 'starting' time. In the light of $\mathrm{CaK}$ we observe the arrival of the 


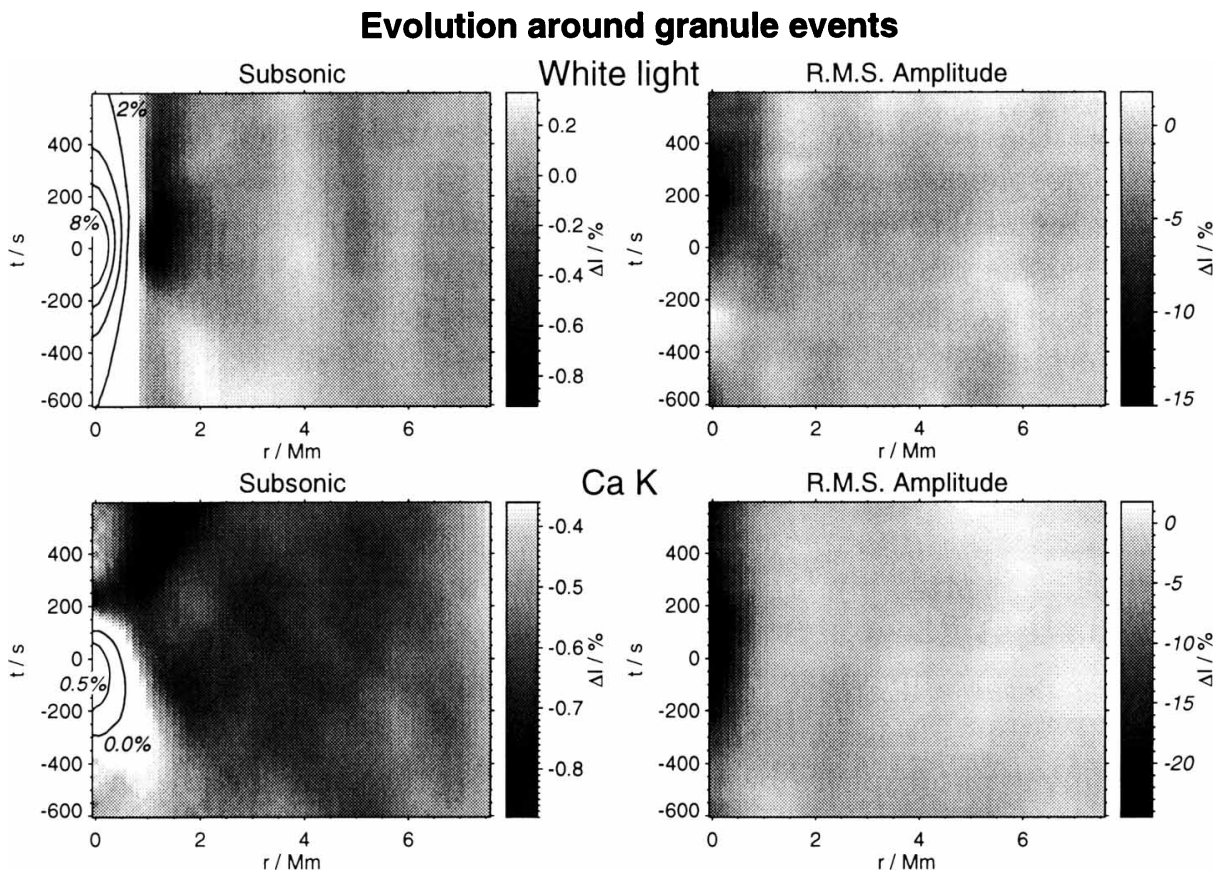

\section{Evolution around intergranule events}

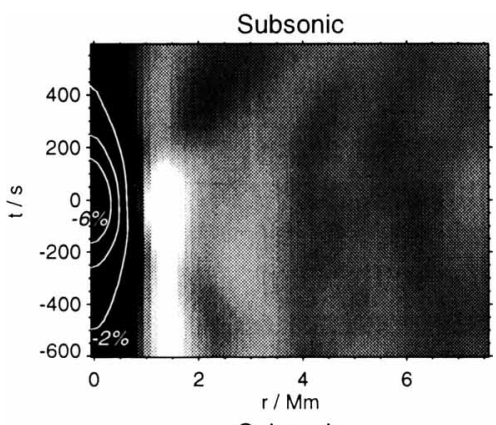

Subsonic

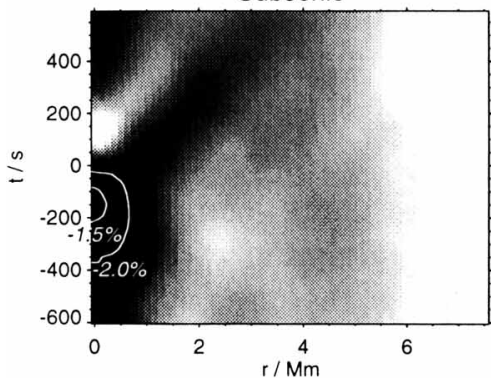

White light

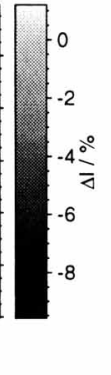

$\mathrm{CaK}$
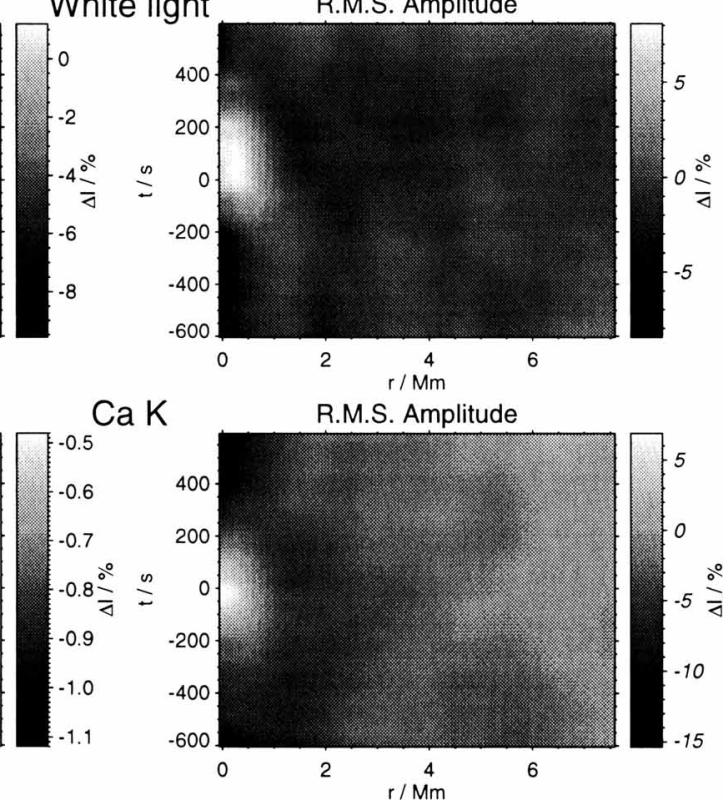

R.M.S. Amplitude

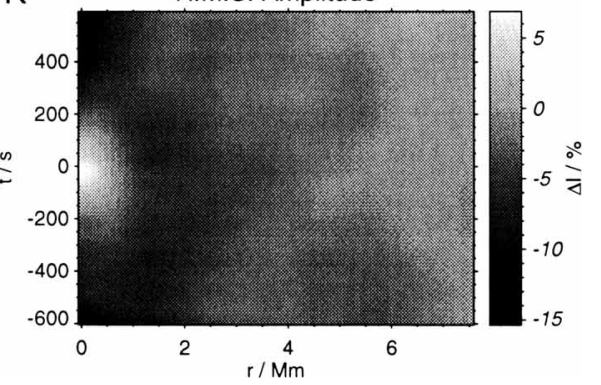

Figure 1. Subsonic intensity and oscillation r.m.s. in photosphere and chromosphere 
front of the disturbance about 200 to $250 \mathrm{sec}$ after the photospheric event. Here, an extended period of increased brightness prevailing at the location of the event is interrupted by a shorter interval $(\sim 100 \mathrm{sec})$ of decreased brightness that we know is associated with upward motion, according to the chromospheric velocity - brightness phase relations discussed e.g. by Hofmann et al. (1996), and by Deubner et al. (1996) in a different context. At this chromospheric level we recognize further that the front of the perturbation expands horizontally, while it also grows in amplitude. With the assumption of an effective height of $\mathrm{CaK}_{232}$ of $1500 \mathrm{~km}$, and with a travel time of $250 \mathrm{sec}$, we derive a velocity of propagation of $\sim 6 \mathrm{~km} \mathrm{~s}^{-1}$, in reasonable agreement with the velocity of sound in the solar atmosphere. The horizontal velocity $\left(\sim 4 \mathrm{~km} \mathrm{~s}^{-1}\right)$ depends on the dispersion relation of the non-radial wave components as well as on the geometry of the source region which is probably far from spherical symmetry; it can therefore not be interpreted without a detailed simulation.

A considerable decrease of power $(10 \%-15 \%)$ is observed at the chromospheric level as well, and we note that the onset of this decrease precedes the one in the photosphere by about 200 sec! At the same instant the photosphere exhibits maximum power, as mentioned above.

Testing Paradigm \#2 (intergranule events): As opposed to the granular case we observe in white light an increase of oscillatory power by $10 \%$ to $15 \%$ in the wake of the event, lasting about 300 sec. Again, the region of increased power is confined approximately within one granular diameter. The power is below average before the event at the source position.

In the light of $\mathrm{CaK}$ we detect the arrival of a perturbation, i.e. an increase by about $1 \%$ in brightness, already about $150 \mathrm{sec}$ after the photospheric event, yielding a vertical velocity of about $8 \mathrm{~km} \mathrm{~s}^{-1}$. As in the granular case, the 'subsonic' component of the perturbation spreads horizontally. Even more prominently we detect in this case the horizontal propagation of a dark precursor (upward motion!) which appears to arise from a pronounced darkening that occurs about $200 \mathrm{sec}$ before the specified event at the same location. The two perturbations are running more or less parallel to each other, the downward motion in the wake of the upward motion on top of the intergranular region.

The oscillatory power increases in the chromosphere again by about $10 \%$. Its maximum coincides with the brightness minimum in the photosphere; however, it precedes the increase of power in the photosphere.

Looking only at the CaK data which were subjected to the 'subsonic' filtering, we note that the two different kinds of sources visible in the photosphere appear to create time dependent brightness patterns in the upper layers that are very nearly negative copies of each other. 


\section{Conclusions}

We have attempted to work out the 2-D response of the solar atmosphere, as observed at two different levels, to a local perturbation induced by impulsive convective motions. We have evaluated the evolution of the two cases, bright photospheric sources ('granules') and dark sources ('intergranular events'), separately, but with the same procedure. Averaging of a large number of events (128) was necessary to isolate the local response from the strong uncorrelated background signal contributed by the global p-mode field.

In either case we recognize the propagation of the photospheric disturbance upon its arrival at the chromospheric level by identifying a distinct local darkening (brightening) with the upward (downward) motion of the arriving pulse. The velocity of propagation in the vertical direction agrees with the sound velocity. It appears to be marginally higher in the second case (intergranule). In either case we find a distinct trace of the horizontal propagation of the disturbance that shows a maximum velocity of about $4 \mathrm{~km} \mathrm{~s}^{-1}$, and originates from the central up(down)flow according to the above identification with the brightness variations. This horizontal wave attains a radial distance of up to $1.5 \mathrm{Mm}$ in our data, and appears most prominent at a slightly smaller distance. The horizontal velocity is consistent with the velocity of propagation of internal gravity waves, and indeed our observations corroborate the theoretical results of Meyer and Schmidt (1967), and the observation of Deubner (1975), who have associated the excitation of gravity waves with the occurrence of strong granules.

Up to this point our findings are symmetric with regard to the polarity of the source (up- or downward pulse). The new observations confront us with puzzling results as this symmetry appears violated: The granular type source causes a decrease of p-mode power in the atmospheric column on top of it (but not next to it), and the flow connected to a strong darkening in the intergranular network causes an increase of similar magnitude. Would wavefront retardation due to quasi-stationary up(down)flow in conjunction with an increase (decrease) of the local sound speed at the source location be sufficient to disperse (concentrate) the ambient p-mode power by a substantial amount, as suggested by Deubner and Laufer (1983)?

Even more alarming appears the observation that the onset, and the maximum of the response of the p-mode power to the perturbation occurs distinctly earlier $(200 \mathrm{sec})$ in $\mathrm{CaK}$ (that is in the upper layer!) than in the continuum, i.e. even earlier $(100-200 \mathrm{sec})$ than the photospheric manifestation of what we have called the source. This finding is compelling evidence that we need to investigate the local response of convective instability in the superadiabatic transition region with regard to the ambient p-mode wave field. 
Regrettably, radial velocity data, which are fundamental for studies of solar atmospheric dynamics, have not been obtained in this investigation. The inferences based on earlier work on 2-D velocity-brightness (V-I) phase differences are helpful, but certainly not satisfactory. With advanced spectroscopic systems that permit either rapid spatial or rapid spectral scanning, the requisite kind of data is gradually becoming available.

Further, the exchange of energy between convective eddies and the atmospheric wave field can be fully understood only on the basis of a quantitative description of the observed phase relations. It is therefore planned to extend our study by including wavelet analyses, that allow the characterization of waveforms together with the determination of the temporal delays that occur between the observed fluctuations.

\section{References}

Deubner. F.-L. (1975) Solar Phys. 40, 333

Deubner, F.-L., Laufer,J. (1983) Solar Phys. 82, 151

Deubner, F.-L., Waldschik, Th., Steffens, S. (1996) Astron. Astrophys. 307, 936

Espagnet, O., Muller, R., Roudier, Th., Mein, P., Mein, N., Malherbe, J.-M. (1996) Astron. Astrophys. 313, 297

Evans, J. W., Michard, R. (1962) ApJ 136, 493

Goode, P. R., Gough, D., Kosovichev, A. (1992) ApJ 387, 707

Hofmann, J., Steffens, S., Deubner, F.-L. (1996) Astron. Astrophys. 308, 192

Kumar, P. (1993) in Seismic Investigation of the Sun and Stars T.Brown ed., ASP Conf. Ser. 42 , p. 15

Kumar, P., Duvall, T. L., Harvey, J. W., Jefferies, S. M., Pomerantz, M. A., Thompson, M. J. (1990) in Proc. Oji International Seminar Y. Osaki and H. Shibahashi eds., Springer Berlin, p. 87

Meyer, F., Schmidt, H. U. (1967) Z. Astrophys. 65, 274

Noyes, R. (1967) in Aerodynamic Phenomena in Stellar Atmospheres R.N.Thomas ed., Acad. Press London, p. 293

Rimmele, T. R., Goode, P. R., Strous, L. H., Stebbins, R. T. (1995) in Proc. of Fourth SOHO Workshop: Helioseismology J.T.Hoeksema, V.Domingo, B.Fleck, B.Battrick eds., ESA SP-376.2, p. 329 